\title{
\begin{tabular}{l|l} 
Mitraries & DSpace@MIT
\end{tabular}
}

\author{
MIT Open Access Articles
}

\section{Scalable Graphene Coatings for Enhanced Condensation Heat Transfer}

The MIT Faculty has made this article openly available. Please share how this access benefits you. Your story matters.

Citation: Preston, Daniel J. et al. “Scalable Graphene Coatings for Enhanced Condensation Heat Transfer." Nano Letters 15.5 (2015): 2902-2909.

As Published: http://dx.doi.org/10.1021/nl504628s

Publisher: American Chemical Society (ACS)

Persistent URL: http://hdl.handle.net/1721.1/107709

Version: Author's final manuscript: final author's manuscript post peer review, without publisher's formatting or copy editing

Terms of Use: Article is made available in accordance with the publisher's policy and may be subject to US copyright law. Please refer to the publisher's site for terms of use. 


\section{Scalable Graphene Coatings for Enhanced}

\section{Condensation Heat Transfer}

Daniel J Preston ${ }^{l}$, Daniela L Mafra ${ }^{2}$, Nenad Miljkovic ${ }^{1,3}$, Jing Kong $^{2}$, and Evelyn N Wang ${ }^{l *}$

${ }^{1}$ Department of Mechanical Engineering, Massachusetts Institute of Technology, Cambridge, Massachusetts 02139, USA

${ }^{2}$ Department of Electrical Engineering and Computer Science, Massachusetts Institute of Technology, Cambridge, Massachusetts 02139, USA

${ }^{3}$ Department of Mechanical Science and Engineering, University of Illinois, Urbana, Illinois 61801, USA

*Corresponding author email: enwang@mit.edu

KEYWORDS: Graphene, condensation, dropwise, heat transfer enhancement, robust, scalable 
ABSTRACT: Water vapor condensation is commonly observed in nature and routinely used as an effective means of transferring heat, with dropwise condensation on non-wetting surfaces exhibiting heat transfer improvement compared to filmwise condensation on wetting surfaces. However, state-of-the-art techniques to promote dropwise condensation rely on functional hydrophobic coatings which either have challenges with chemical stability or are so thick that any potential heat transfer improvement is negated due to the added thermal resistance of the coating. In this work, we show the effectiveness of ultra-thin scalable chemical vapor deposited (CVD) graphene coatings to promote dropwise condensation while offering robust chemical stability and maintaining low thermal resistance. Heat transfer enhancements of $4 \mathrm{x}$ were demonstrated compared to filmwise condensation, and the robustness of these CVD coatings was superior to typical hydrophobic monolayer coatings. Our results indicate that graphene is a promising surface coating to promote dropwise condensation of water in industrial conditions, with the potential for scalable application via CVD. 
Graphene is a two-dimensional material composed of carbon atoms arranged in a hexagonal lattice which has received significant attention since 2004 due to its unique and remarkable physical properties. ${ }^{1}$ Prominent examples of the applicability of graphene include electronic device interconnects due to high charge carrier mobility, ${ }^{2}$ transparent electrodes for solar cell devices, ${ }^{3}$ and membranes for water desalination. ${ }^{4}$ Graphene has also been used in thermal management applications due to its ability to improve device thermal conductivity and spread heat. ${ }^{5}$ However, with graphene being a relatively new material, many applications have not yet been thoroughly explored.

One such application is the promotion of dropwise condensation. In typical industrial systems, condensed vapor forms a thin liquid film on the condenser surface due to the high surface energy associated with the majority of industrial heat exchanger materials (i.e., clean metals and metal oxides). This mode, known as filmwise condensation, is not desired due to the large thermal resistance to heat transfer. ${ }^{6}$ Conversely, on low surface energy materials, the condensed vapor forms discrete liquid droplets. During this dropwise mode of condensation, droplets roll off at sizes approaching the capillary length $(\approx 2 \mathrm{~mm}$ for water) and clear the surface for re-nucleation, commonly resulting in 5-7x higher heat transfer performance compared to filmwise condensation. $^{7}$

Dropwise condensation is typically achieved by functionalizing the condenser surface with a hydrophobic coating, for example, a fluorocarbon monolayer, wax, or polymer. ${ }^{7 a}{ }^{8}$ Monolayer coatings $(\approx 1 \mathrm{~nm}$ thick) of long-chain fluorocarbons or fatty acids can induce hydrophobicity with negligible added thermal resistance, but are often not robust, i.e., chemically stable, over extended periods of time and therefore unsuitable in industrial applications. ${ }^{7 a, 9}$ Thicker polymer coatings $(>1 \mu \mathrm{m})$ such as PTFE have shown the potential to maintain robust hydrophobicity, but 
typically have a large thermal resistance that can negate the heat transfer enhancement gained by promoting dropwise condensation. ${ }^{7 a}$ More recently developed methods such as initiated chemical vapor deposition (iCVD) and plasma enhanced chemical vapor deposition (PECVD) have been used to grow ultra-thin $(<40 \mathrm{~nm})$ conformal polymer coatings with success in achieving dropwise condensation..$^{9 b, 10}$ However, the durability of iCVD and PECVD coatings requires further characterization due to limited extended testing to assess mechanical wear and chemical stability for long term condensing applications.

Meanwhile, graphene displays hydrophobic behavior ${ }^{11}$, and its inert chemical nature and demonstrated mechanical strength suggest that it will resist degradation under typical condenser conditions. ${ }^{3,12}$ Furthermore, the thermal resistance of a graphene coating is well-characterized ${ }^{13}$ and is negligible in condensation applications (see Supporting Information, section S2), and it can be applied relatively scalably via CVD. ${ }^{14}$ Although graphene was initially suggested to have complete wetting transparency ${ }^{15}$, its hydrophobic nature has since been elucidated through

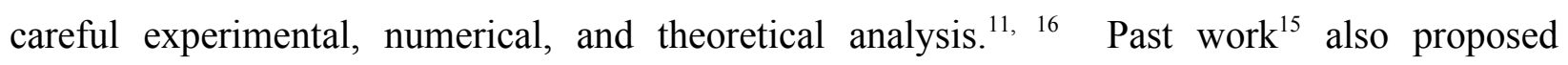
graphene coatings to promote dropwise condensation, but the results of the experimental analysis did not show the expected improvement in heat transfer compared to filmwise condensation. The presence of non-condensible gases in the experimental setup not only reduced the improvement gained by promoting dropwise condensation to $30-40 \%$ as opposed to $500-700 \%$, but also resulted in reported condensation heat transfer coefficients three orders of magnitude lower than typical values without noncondensible gases. ${ }^{7 a}$ Furthermore, the mechanism for the dropwise condensation behavior was attributed to the "transparent" graphene layer protecting the copper from oxidation and preserving the intrinsic hydrophobic behavior of copper, while it has 
been demonstrated that copper is actually intrinsically hydrophilic ${ }^{17}$ like other high-surfaceenergy materials. ${ }^{18}$

In this work, we demonstrate the uniform coating of high-purity (>99.99\%) copper with graphene by both low and atmospheric pressure CVD. Both the low-pressure CVD (LPCVD) and the atmospheric pressure CVD (APCVD) graphene were single-layer. Subsequently, we experimentally demonstrated a $4 \mathrm{x}$ higher heat transfer coefficient for dropwise condensation of water on copper coated by graphene (both LPCVD and APCVD) compared to filmwise condensation on bare copper, in good agreement with theoretical models used for each case. The robustness of these graphene coatings was compared to a long-chain fluorocarbon monolayer commonly used to promote dropwise condensation, where $100^{\circ} \mathrm{C}$ steam was condensed on both samples continuously. The fluorocarbon monolayer coating degraded completely in under 12 hours, while for the graphene coatings, dropwise condensation was observed over a two-week span without showing signs of degradation. These results suggest that graphene is a robust and nonreactive coating material which enhances condensation heat transfer by promoting dropwise condensation.

The copper samples used as substrates for graphene CVD were high purity tubes $(>99.99 \%$, $\mathrm{OD}=1 / 4$ inch, McMaster-Carr $)$ and sections of sheet metal $(>99.99 \%$, thickness $=0.032 \mathrm{inch}$, McMaster-Carr). Prior to the CVD process, the copper samples were sonicated in acetone, triple-rinsed with deionized water, submersed in $2.0 \mathrm{M}$ hydrochloric acid, again triple-rinsed with deionized water, and finally treated for 10 minutes with argon plasma (Harrick PDC-001), which removes hydrocarbons via physical bombardment. ${ }^{19}$ This process was also used to clean the bare copper samples for characterization of filmwise condensation under ideal conditions 
since past work has shown that bare copper exhibits filmwise condensation during continuous condensation. ${ }^{20}$

The CVD process was then performed on the copper samples at both low and atmospheric pressure in a 1-inch quartz tube furnace. For both processes, the furnace was heated to $1000{ }^{\circ} \mathrm{C}$, and hydrogen gas (with a flow rate of $10 \mathrm{secm}$ ) for the LPCVD process and argon gas (with a flow rate of $500 \mathrm{sccm}$ ) for APCVD process flowed over the samples for 30 min prior to graphene growth. Methane gas was then introduced, and the synthesis of graphene was performed over 30 min at 1.9 Torr and atmospheric pressure for the LPCVD (with a flow rate of $\mathrm{H}_{2} / \mathrm{CH}_{4}=70 / 4$ sccm) and APCVD (with a flow rate of $\mathrm{Ar} / \mathrm{CH}_{4}=500 / 3 \mathrm{sccm}$ ) coatings, respectively. ${ }^{21}$ Finally, the samples were cooled to room temperature under hydrogen $(10 \mathrm{sccm}) /$ argon gas $(500 \mathrm{sccm})$ for LPCVD/APCVD and then exposed to laboratory air (i.e., non-filtered). While exposure to air leaves the possibility for contamination, the difference in wettability is expected to be negligible across different laboratory environments as indicated in past work ${ }^{18 b}$ (Further characterization specifically for graphene contamination under several different environments is an important topic and should be investigated in future research).

We characterized the samples using field-emission scanning electron microscopy (Zeiss UltraPlus) to determine the surface morphology, shown in Figure 1. Copper grains are visible on both the LPCVD and APCVD surfaces, and it was observed that the graphene covered the entire surface of the copper. (The absence of visible copper grains on the bare copper surface is attributed to not heating the bare copper to $1000{ }^{\circ} \mathrm{C}$ as is required during graphene CVD.) Further characterization of the samples was conducted with Raman spectroscopy using a confocal Raman microscope with a $532 \mathrm{~nm}$ laser. Representative Raman spectra for the CVD graphene coatings after transfer onto a silicon substrate are shown in Figure 1(e), where the ratio 
of 2D:G peaks $(4-5 \mathrm{x})$ and the full width at half maximum of the $2 \mathrm{D}$ peak $\left(25-30 \mathrm{~cm}^{-1}\right)$ demonstrate the presence of single-layer graphene for both CVD methods. Optical characterization of the CVD graphene after transfer onto a transparent substrate (see Supporting Information) was also performed to study the graphene thickness over a larger area than the Raman laser spot size (Raman spot size is $\sim 1 \mu \mathrm{m}$ while the transmittance measurement spot size is $\sim 5 \mathrm{~mm})$. The optical characterization indicated that both the LPCVD and APCVD graphene were predominately single-layer. ${ }^{22}$

We determined the surface wetting properties for water by goniometric characterization, where the advancing and receding contact angles in Figure 2 describe the surface wettability. ${ }^{23}$ Both the advancing and receding contact angles need to be considered to determine the force which holds a droplet stationary on an inclined condensing surface against the force of gravity, which directly affects droplet departure size and condensation heat transfer. ${ }^{24}$ The average advancing/receding contact angles were $87 \pm 5^{\circ} / 64 \pm 5^{\circ}$ for the LPCVD graphene and $93 \pm 5^{\circ} / 56 \pm 5^{\circ}$ for the APCVD graphene, determined from six points on each sample using a piezoelectric picoliter-scale droplet dispenser microgoniometer (Kyowa MCA-3) with the receding contact angle obtained during droplet evaporation and observed to exhibit constant receding contact angle behavior. ${ }^{25}$ The contact angle hysteresis was attributed to sporadic defects on the surface, possibly at graphene grain boundaries. The droplet departure size during water vapor condensation, defined as the diameter at which droplets begin to slide down the condenser wall, is shown in Figure 2(c,f). The average droplet departure diameters were $2.4 \pm 0.1 \mathrm{~mm}$ on LPCVD graphene compared to $2.8 \pm 0.1 \mathrm{~mm}$ on APCVD graphene, suggesting that the condensation heat transfer coefficient on LPCVD graphene will be slightly higher than on APCVD graphene as droplets shed at smaller sizes and refresh the surface for re-nucleation. The 
contact angle on the clean bare copper surface was $\approx 0^{\circ}$, with no distinction between advancing and receding.

We experimentally obtained the overall heat transfer performance of the graphene-coated copper tubes in a controlled vacuum chamber. Prior to condensation experiments, the vacuum chamber was evacuated to a pressure of $P<1.0 \mathrm{~Pa}$ to eliminate the presence of noncondensable gases, which have been shown to severely degrade condensation heat transfer performance. ${ }^{26}$ Water vapor was then introduced from a canister of degassed, deionized water attached to the vacuum chamber. The copper tube temperature was regulated by an internal chiller water flow loop which was isolated from the interior of the vacuum chamber, and the heat transfer through the tube wall was determined as a function of the chiller water flow rate and chiller water temperature at the tube inlet and outlet. As the copper tube was chilled internally, water vapor within the chamber condensed on the outer tube surface. The water vapor pressure within the chamber was maintained at values ranging from 2 to $5 \mathrm{kPa}$ (corresponding to saturated water temperatures of 17 to $33{ }^{\circ} \mathrm{C}$ ), which are typical for industrial condenser applications. ${ }^{27}$

Photographs of condensation on the exterior tube surfaces are shown in Figure 3. The bare copper tube underwent filmwise condensation of water vapor regardless of the temperature difference between the tube and the surrounding water vapor due to the spreading nature of water on clean copper (Figure 3(a,b)). The LPCVD (Figure 3(c,d)) and APCVD (Figure 3(e,f)) graphene coated tubes have visible graphene layers when dry (Figure 3(c,e)), and these coated tubes exhibited dropwise condensation over the full range of experimental conditions.

The overall heat flux, determined by the change in sensible heat of the chiller water, was obtained along with the log mean temperature difference (LMTD) between the chiller water and the temperature corresponding to the pressure of the surrounding water vapor for the bare copper 
(diamonds), LPCVD graphene (squares), and APCVD graphene (circles) (Figure 4(a)). The overall heat flux increased monotonically with the LMTD, where the local slope of this curve represents the overall heat transfer coefficient. Figure 4(b) shows the condensation heat transfer coefficient extracted from the overall heat transfer coefficient (see Supporting Information) as a function of vapor pressure while holding the supersaturation $S=P_{\text {vapor }} / P_{\text {sat }}\left(T_{\text {wall }}\right)$ constant $(S=$ 1.2). The theoretical predictions (dashed curves) were obtained from the droplet growth and distribution model for the graphene-coated condenser and from the Nusselt model for filmwise condensation on the bare copper condenser, and were in good agreement with the experimental data (for model derivation and parameters, see Supporting Information). The assumption of uniform wall temperature for the models was justified because the temperature variation in the chiller water from the inlet to outlet of the sample was over an order of magnitude less than the temperature difference from the sample to the surrounding vapor. The condensation heat transfer coefficient for the LPCVD and APCVD graphene coated copper tubes $\left(\approx 60 \pm 20 \mathrm{~kW} / \mathrm{m}^{2} \mathrm{~K}\right)$ was $4 \mathrm{x}$ greater than that measured for filmwise condensation on bare copper $\left(\approx 15 \pm 9 \mathrm{~kW} / \mathrm{m}^{2} \mathrm{~K}\right)$. Note that the dropwise condensation heat transfer coefficient decreases at low subcooling because the interfacial heat transfer coefficient becomes a major resistance to heat transfer, ${ }^{7 a, 8 e, 28}$ while the filmwise condensation heat transfer coefficient increases at low subcooling as the film

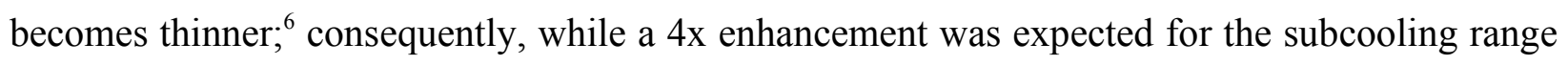
used to characterize the heat transfer coefficient in the present work $(3.5-5 \mathrm{~K})$, the typically reported 5-7x heat transfer coefficient enhancement ${ }^{7 \mathrm{a}}$ would be realized at higher subcooling (over $\approx 10 \mathrm{~K}$, see Supporting Information, Section S9).

The error for the condensation heat transfer coefficient was determined by propagating uncertainties associated with the chiller water thermocouples and mass flow meter, the pressure 
sensor inside the chamber, the sample surface area, and the Gnielinski correlation for heat transfer from the chiller water bulk to the internal surface of the sample. Since the condensation heat transfer coefficient, $h c$, is not simply a function of a product of powers, the error must be determined from the first partial derivatives of $h_{c}$ with respect to its components along with the uncertainties of its components (described in detail in the Supporting Information, Section S5).

In addition to improved condensation heat transfer by promoting dropwise condensation, graphene coatings also exhibit inert chemical behavior and excellent mechanical strength, which are expected to result in improved resistance to routine wear during condensation and provide a robust alternative to current state-of-the-art dropwise functionalization coatings. For direct comparison, a monolayer coating of trichloro $(1 \mathrm{H}, 1 \mathrm{H}, 2 \mathrm{H}, 2 \mathrm{H}$-perfluorooctyl)silane (TFTS) was applied to a clean copper sample and, along with the graphene-coated samples, underwent an accelerated endurance test which consisted of continuous condensation of $100{ }^{\circ} \mathrm{C}$ steam. The TFTS coating was applied via vapor-phase deposition, where the copper substrate was cleaned as described for the graphene CVD, but oxygen plasma was substituted for argon plasma. The copper substrate was then placed in a dessicator immediately following the oxygen plasma treatment along with a vial containing $2 \mathrm{~mL}$ of TFTS. The dessicator was evacuated with a vacuum pump for 90 seconds, after which the sample was left in the TFTS vapor for 10 minutes. The sample was then removed from the dessicator, solvent rinsed, and dried with a clean nitrogen stream. The advancing/receding contact angles were $120 \pm 5 / 82 \pm 5^{\circ}$ on the TFTS-coated copper sample as fabricated.

The endurance test was performed in a controlled positive-pressure continuous condensation chamber (see Supporting Information, section S7). The samples were cooled to a surface temperature of $95 \pm 1^{\circ} \mathrm{C}$ and exposed to a continuous supply of $100{ }^{\circ} \mathrm{C}$ steam provided from a 
reservoir of degassed, deionized water. Initially, dropwise condensation was observed on both the LPCVD and APCVD graphene coated samples and the TFTS coated sample (first column of Figure 5). However, the TFTS coating degraded noticeably within the first $30 \mathrm{~min}$ of testing, with decreased advancing and receding contact angles observed, and completely transitioned to filmwise condensation within 12 hours (Figure 5(c)), likely due to stripping of the coating by oxidation (X-ray photoelectron spectroscopy (K-Alpha) revealed that only 3\% of the originally observed atomic percent of fluorine, a primary component of TFTS, remained on the surface after the continuous condensation experiment). ${ }^{9 c,}{ }^{29}$ Conversely, the LPCVD and APCVD graphene coated samples both sustained dropwise condensation for over two weeks with no signs of degradation when the experiments were discontinued (Figure 5(a,b)). Additionally, these graphene coatings can be altered to multi-layer graphene or even single-/multi-layer graphene composites $^{30}$ without drastic effect on the heat transfer (since the added thermal resistance is negligible, see Supporting Information Section S2) and can thus potentially be tailored to better prevent oxidation if it poses a problem. Further discussion on graphene's chemical robustness is presented in the Supporting Information, Section S8.

While graphene offers a robust coating material to promote dropwise condensation on industrial metals, it is not a likely candidate to induce superhydrophobic behavior on micro- and nanostructured materials due to its relatively low advancing and receding contact angles compared to fluoropolymer coatings typically used for this application. While this eliminates the ability of graphene-coated surfaces to promote jumping droplet condensation, ${ }^{31}$ the improvement in heat transfer coefficient of $4 \mathrm{x}$ provided by dropwise-promoting graphene coatings compared to filmwise condensation outweighs the marginal additional increase of $30-40 \%$ gained by jumping droplet condensation compared to dropwise condensation. Another potential limitation 
of these graphene coatings is their inability to induce dropwise condensation in systems which use low-surface-tension working fluids such as pentane; these fluids are expected to exhibit low contact angles and spread on graphene-coated surfaces, resulting in filmwise condensation.

This study demonstrates that graphene CVD coatings are a viable method to promote dropwise condensation of water in industrial conditions, with a demonstrated improvement in heat transfer performance of $4 \mathrm{x}$ compared to clean industrial metals and superior robustness compared to state-of-the-art dropwise-promoting monolayer coatings as demonstrated under continuous condensation of $100{ }^{\circ} \mathrm{C}$ steam. This result promises significant energy savings in applications such as water harvesting, thermal management, industrial power generation, and building heating and cooling. 

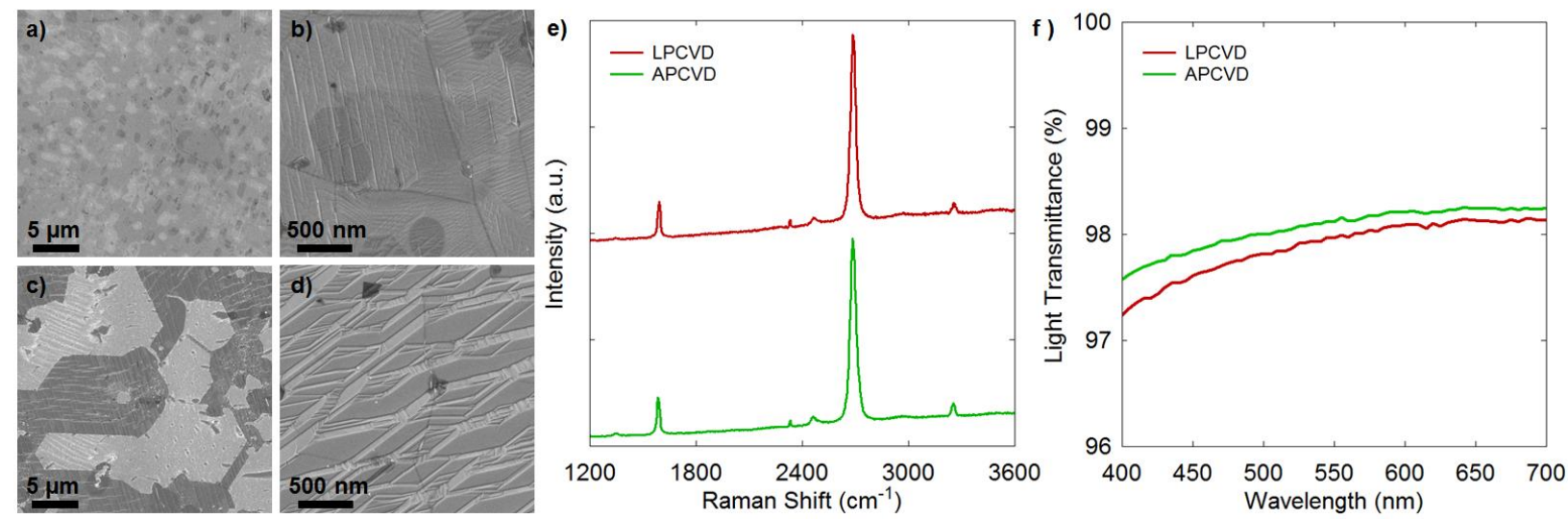

Figure 1. Field emission scanning electron microscopy images for $(a, b)$ the low-pressure CVD graphene coating and $(\mathrm{c}, \mathrm{d})$ the atmospheric pressure CVD graphene coating on high-purity (> 99.99\%) copper substrates. During growth, the native copper oxide layer is reduced by $\mathrm{H}_{2}$ gas at high temperature, and the underlying copper forms pronounced grains. Upon exposure to a $\mathrm{CH}_{4}$ at $1000{ }^{\circ} \mathrm{C}$, graphene islands nucleate and grow over the surface until colliding with other islands. The copper grains remain visible. (e) Representative Raman spectra for the CVD graphene layers after transfer to a silicon substrate, obtained with a confocal Raman microscope using a $532 \mathrm{~nm}$ laser, demonstrate the presence of single-layer graphene for both CVD methods. (f) Optical characterization of the graphene transferred onto a transparent substrate indicated that both the LPCVD and APCVD graphene were predominately single-layer. ${ }^{22}$ 

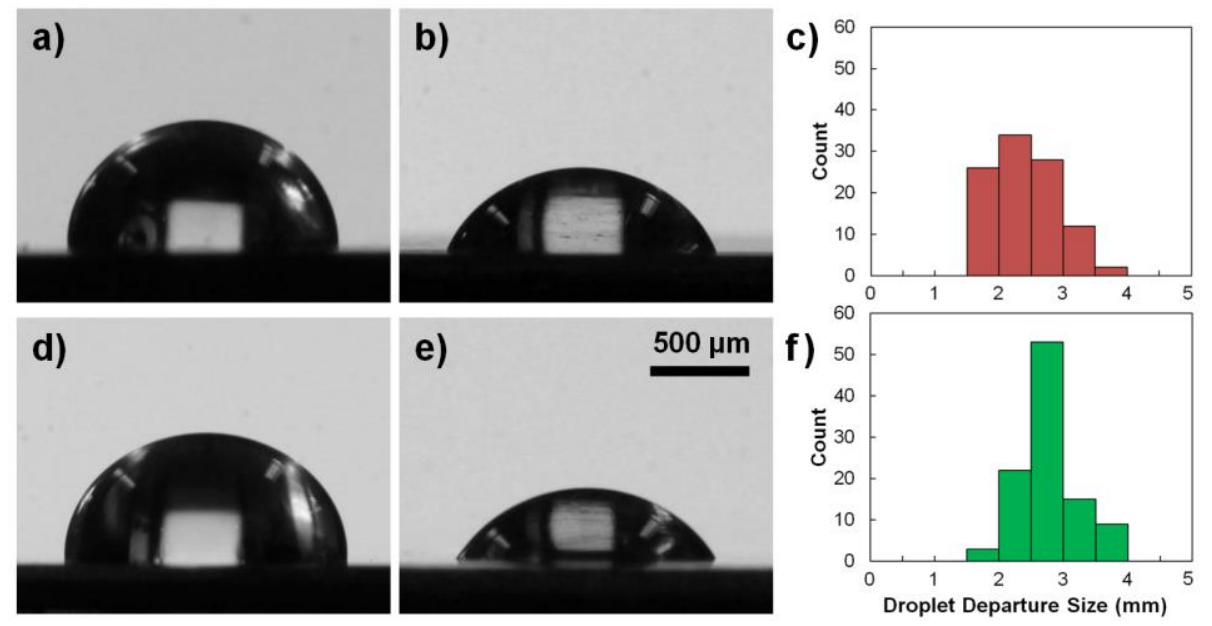

Figure 2. Representative images of (a) advancing and (b) receding contact angles of water on LPCVD graphene grown on a high-purity copper substrate, obtained by goniometric measurement. A histogram of droplet departure size during water condensation on LPCVD graphene is shown in (c). The advancing and receding contact angle for water on APCVD graphene are shown in (d) and (e), respectively, and a histogram of droplet departure size during water condensation on APCVD graphene is shown in (f). The average droplet departure diameter during water condensation on APCVD graphene is $2.8 \pm 0.1 \mathrm{~mm}$ compared to $2.4 \pm 0.1$ mm on LPCVD graphene, suggesting a slightly higher expected condensation heat transfer coefficient on LPCVD graphene than on APCVD graphene. 

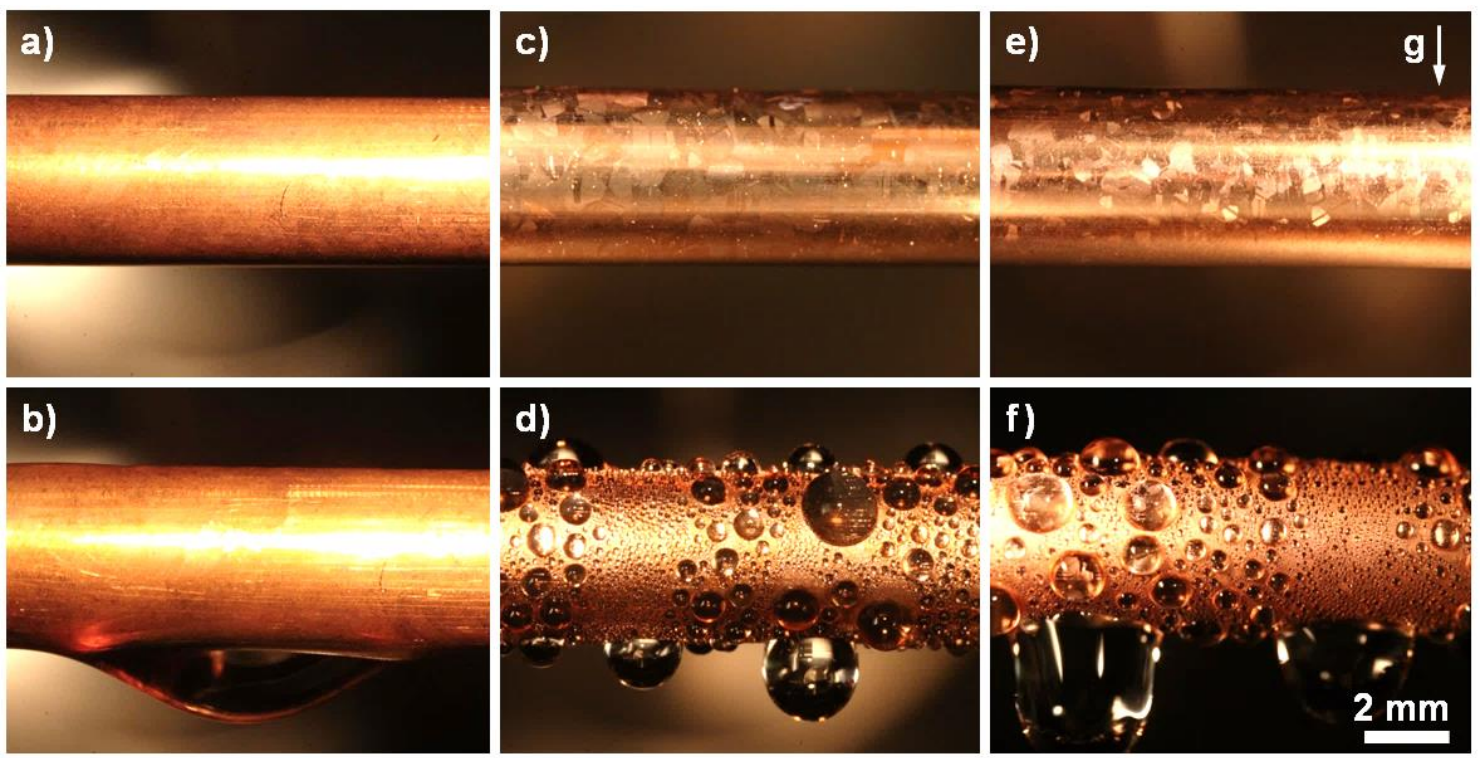

Figure 3. Photographs of a clean high-purity copper condenser tube (a) under vacuum and (b) undergoing filmwise condensation of deionized and degassed water vapor in the experimental vacuum chamber. Similarly, photographs of the graphene-coated high-purity copper condenser tubes are shown under vacuum and undergoing dropwise condensation of water, with the LPCVD graphene coating in (c) and (d) and the APCVD graphene coating in (e) and (f). 

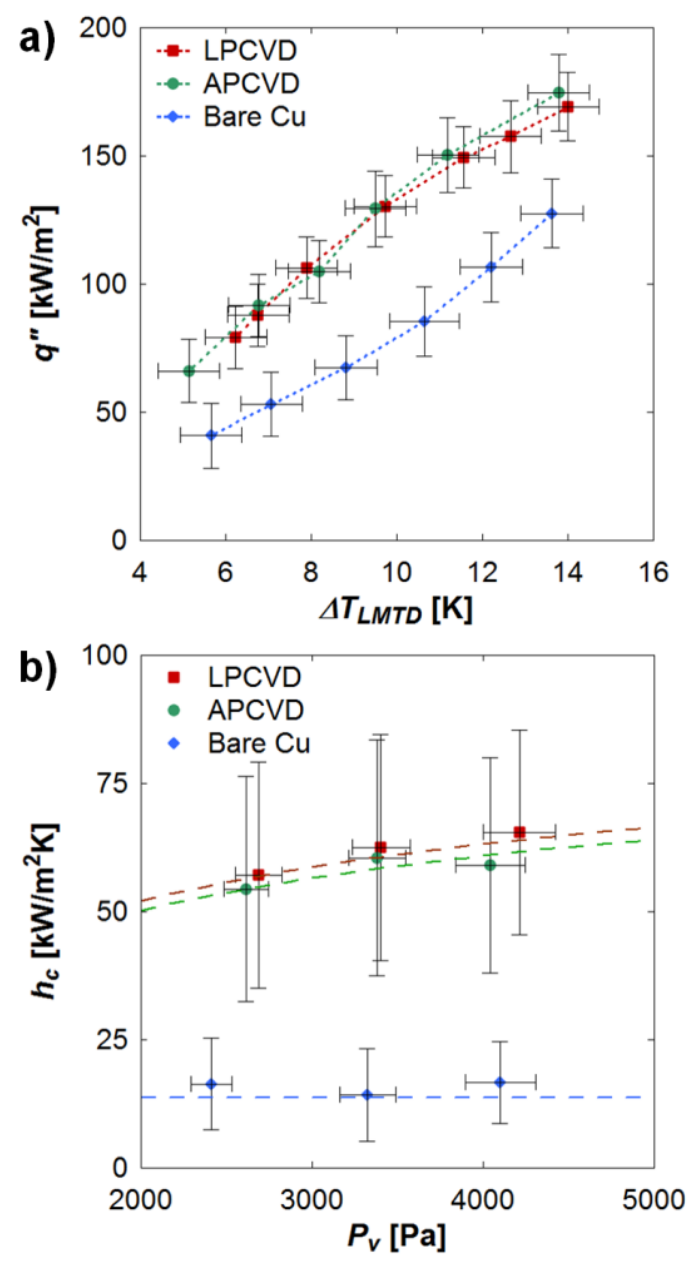

Figure 4. Water condensation heat transfer performance for the copper tubes with and without graphene coatings. The graphene-coated condensers exhibit dropwise condensation, while the bare copper undergoes filmwise condensation. Overall surface heat flux $(q$ ”) is shown as a function of the steady state experimental chiller-water-to-vapor log mean temperature difference $\left(\triangle T_{L M T D}\right)$ in (a), where the slope of the data trend represents the overall heat transfer coefficient, that is, the combination of the chiller water flow, copper tube, graphene coating (for coated tubes), and condensation heat transfer coefficients. (b) Experimental (points) and theoretical (dashed curves) steady-state condensation heat transfer coefficient $\left(h_{c}\right)$, which includes graphene coatings where applicable, as a function of surrounding saturated vapor pressure $\left(P_{v}\right)$. Error bars 
indicate the propagation of error associated with the fluid inlet and outlet temperature differential $( \pm 0.2 \mathrm{~K})$ and pressure measurement $( \pm 2.5 \%)$. Theoretical predictions were obtained from the droplet growth and distribution model for the graphene-coated condensers with droplet departure size as an input parameter (for model derivation and parameters, see Supporting Information) and from the Nusselt model for filmwise condensation on the bare copper condenser. 


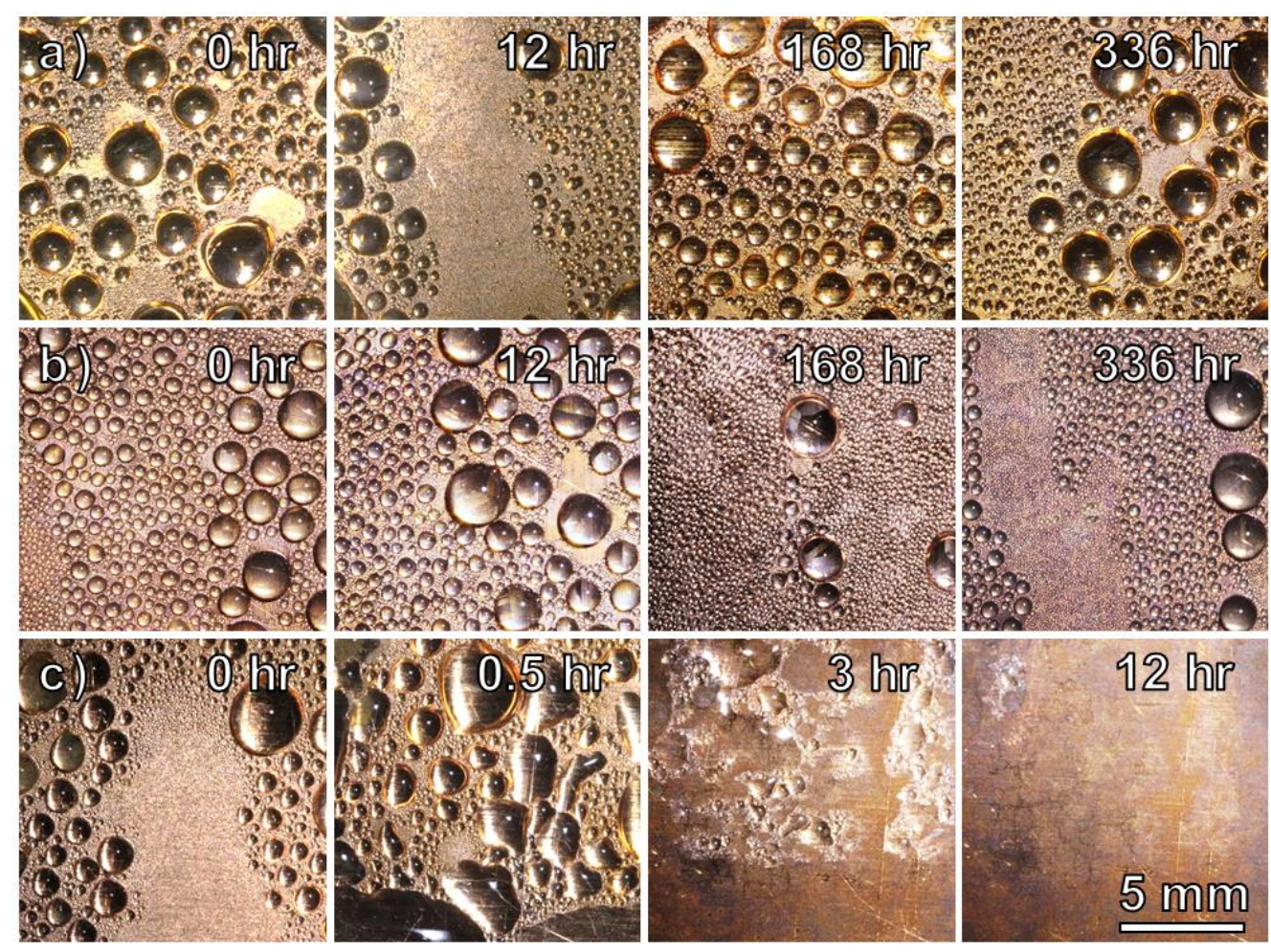

Figure 5. Time-lapse images of continuous condensation of $100^{\circ} \mathrm{C}$ steam on (a) LPCVD, (b) APCVD, and (c) TFTS coatings on high-purity copper samples. The robust promotion of dropwise condensation by the graphene coatings is investigated over two weeks without showing signs of degradation, in contrast with the TFTS coating, which degraded and transitioned to filmwise condensation in less than 12 hours. 


\section{ASSOCIATED CONTENT}

Supporting Information. Supplementary material is provided for ESEM characterization of the CVD graphene coatings, coating thermal resistance scaling, operation of the controlled condensation chamber for heat transfer measurement, calculation of condensation heat transfer coefficient and error analysis, modeling of condensation heat transfer coefficient, and operation of the robustness characterization setup. This material is available free of charge via the Internet at http://pubs.acs.org.

\section{AUTHOR INFORMATION}

\section{Corresponding Author}

*Email: enwang@mit.edu

\section{Author Contributions}

E.N.W., J.K., D.J.P, and N.M. conceived the initial idea of this research. E.N.W. and J.K guided the work. D.L.M. and D.J.P. fabricated, functionalized, and characterized the experimental samples. D.J.P. carried out the experiments and collected and analyzed the data. D.J.P. and N.M. carried out the theoretical analysis. All authors were responsible for writing the paper and have given approval to the final version of the manuscript.

\section{ACKNOWLEDGMENT}

We gratefully acknowledge funding support from the Office of Naval Research (ONR) with Dr. Mark Spector as program manager. We also acknowledge the support from the National Science Foundation through the Major Research Instrumentation Grant for Rapid Response Research (MRI-RAPID) for the microgoniometer. D.J.P. acknowledges funding received by the National 
Science Foundation Graduate Research Fellowship under Grant No. 1122374. Any opinion, findings, conclusions, or recommendations expressed in this material are those of the authors(s) and do not necessarily reflect the views of the National Science Foundation. D.L.M. acknowledges the Brazilian agency CNPq. J.K. acknowledges the support through the STC Center for Integrated Quantum Materials from NSF (US) grant DMR-1231319. This work was performed in part at the Center for Nanoscale Systems (CNS), a member of the National Nanotechnology Infrastructure Network (NNIN), which is supported by the National Science Foundation under NSF award no. ECS-0335765. CNS is part of Harvard University.

\section{ABBREVIATIONS}

CVD, chemical vapor deposition; APCVD, atmospheric pressure CVD; LPCVD, low pressure CVD; FESEM, field emission scanning electron microscope; ESEM, environmental scanning electron microscope.

Table of Contents Graphic:

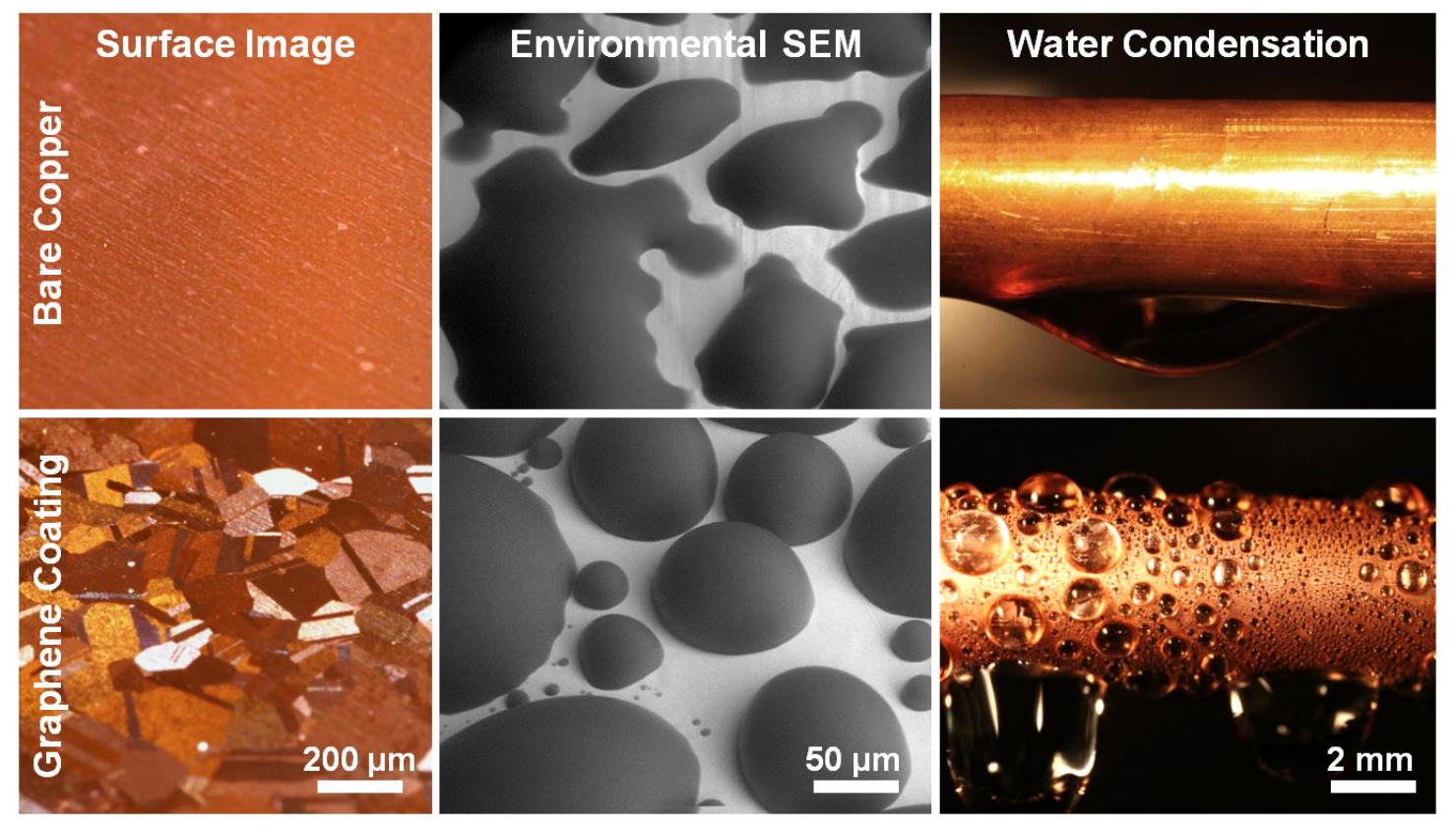




\section{REFERENCES}

1. (a) Novoselov, K. S.; Jiang, D.; Schedin, F.; Booth, T. J.; Khotkevich, V. V.; Morozov, S. V.; Geim, A. K., Two-dimensional atomic crystals. P Natl Acad Sci USA 2005, 102 (30), 1045110453; (b) Novoselov, K. S.; Geim, A. K.; Morozov, S. V.; Jiang, D.; Zhang, Y.; Dubonos, S. V.; Grigorieva, I. V.; Firsov, A. A., Electric field effect in atomically thin carbon films. Science 2004, 306 (5696), 666-669.

2. Chen, X. Y.; Akinwande, D.; Lee, K. J.; Close, G. F.; Yasuda, S.; Paul, B. C.; Fujita, S.; Kong, J.; Wong, H. S. P., Fully Integrated Graphene and Carbon Nanotube Interconnects for Gigahertz High-Speed CMOS Electronics. Ieee T Electron Dev 2010, 57 (11), 3137-3143.

3. (a) Li, X. S.; Zhu, Y. W.; Cai, W. W.; Borysiak, M.; Han, B. Y.; Chen, D.; Piner, R. D.; Colombo, L.; Ruoff, R. S., Transfer of Large-Area Graphene Films for High-Performance Transparent Conductive Electrodes. Nano Lett 2009, 9 (12), 4359-4363; (b) Wang, X.; Zhi, L. J.; Mullen, K., Transparent, conductive graphene electrodes for dye-sensitized solar cells. Nano Lett 2008, $8(1), 323-327$.

4. (a) Humplik, T.; Lee, J.; O'Hern, S. C.; Fellman, B. A.; Baig, M. A.; Hassan, S. F.; Atieh, M. A.; Rahman, F.; Laoui, T.; Karnik, R.; Wang, E. N., Nanostructured materials for water desalination. Nanotechnology 2011, 22 (292001); (b) O'Hern, S. C.; Stewart, C. A.; Boutilier, M. S. H.; Idrobo, J. C.; Bhaviripudi, S.; Das, S. K.; Kong, J.; Laoui, T.; Atieh, M.; Karnik, R., Selective Molecular Transport through Intrinsic Defects in a Single Layer of CVD Graphene. Acs Nano 2012, 6 (11), 10130-10138.

5. (a) Goli, P.; Ning, H.; Li, X. S.; Lu, C. Y.; Novoselov, K. S.; Balandin, A. A., Thermal Properties of Graphene-Copper-Graphene Heterogeneous Films. Nano Lett 2014, 14 (3), 1497 1503; (b) Malekpour, H.; Chang, K. H.; Chen, J. C.; Lu, C. Y.; Nika, D. L.; Novoselov, K. S.; Balandin, A. A., Thermal Conductivity of Graphene Laminate. Nano Lett 2014, 14 (9), 51555161; (c) Yan, Z.; Liu, G. X.; Khan, J. M.; Balandin, A. A., Graphene quilts for thermal management of high-power GaN transistors. Nat Commun 2012, 3 (827).

6. Nusselt, W., The surface condensation of water vapour. Z Ver Dtsch Ing 1916, 60, 541546.

7. (a) Rose, J. W., Dropwise condensation theory and experiment: a review. P I Mech Eng a-J Pow 2002, 216 (A2), 115-128; (b) Schmidt, E.; Schurig, W.; Sellschopp, W., Condensation of water vapour in film- and drop form. Z Ver Dtsch Ing 1930, 74, 544-544. 
8. (a) Das, A. K.; Kilty, H. P.; Marto, P. J.; Andeen, G. B.; Kumar, A., The use of an organic self-assembled monolayer coating to promote dropwise condensation of steam on horizontal tubes. J Heat Trans-T Asme 2000, 122 (2), 278-286; (b) Marto, P. J.; Looney, D. J.; Rose, J. W.; Wanniarachchi, A. S., Evaluation of Organic Coatings for the Promotion of Dropwise Condensation of Steam. Int J Heat Mass Tran 1986, 29 (8), 1109-1117; (c) Vemuri, S.; Kim, K. J., An experimental and theoretical study on the concept of dropwise condensation. Int J Heat Mass Tran 2006, 49 (3-4), 649-657; (d) Vemuri, S.; Kim, K. J.; Wood, B. D.; Govindaraju, S.; Bell, T. W., Long term testing for dropwise condensation using self-assembled monolayer coatings of n-octadecyl mercaptan. Appl Therm Eng 2006, 26 (4), 421-429; (e) Enright, R.; Miljkovic, N.; Alvarado, J. L.; Kim, K. J.; Rose, J. W., Dropwise Condensation on Micro- and Nanostructured Surfaces. Nanoscale and Microscale Thermophysical Engineering 2014, 18 (3), 223-250.

9. (a) Boinovich, L. B.; Emelyanenko, A. M., Hydrophobic materials and coatings: Principles of design, properties and applications. Usp Khim + 2008, 77 (7), 619-638; (b) Paxson, A. T.; Yague, J. L.; Gleason, K. K.; Varanasi, K. K., Stable Dropwise Condensation for Enhancing Heat Transfer via the Initiated Chemical Vapor Deposition (iCVD) of Grafted Polymer Films. Adv Mater 2013, 26 (3), 418-423; (c) Holden, K. M.; Wanniarachchi, A. S.; Marto, P. J.; Boone, D. H.; Rose, J. W., The Use of Organic Coatings to Promote Dropwise Condensation of Steam. J Heat Trans-T Asme 1987, 109 (3), 768-774.

10. Miljkovic, N.; Preston, D. J.; Enright, R.; Wang, E. N., Electrostatic charging of jumping droplets. Nat Commun 2013, 4 (2517).

11. (a) Raj, R.; Maroo, S. C.; Wang, E. N., Wettability of Graphene. Nano Lett 2013, 13 (4), 1509-1515; (b) Shin, Y. J.; Wang, Y. Y.; Huang, H.; Kalon, G.; Wee, A. T. S.; Shen, Z. X.;

Bhatia, C. S.; Yang, H., Surface-Energy Engineering of Graphene. Langmuir 2010, 26 (6), 37983802.

12. (a) Li, X. L.; Zhang, G. Y.; Bai, X. D.; Sun, X. M.; Wang, X. R.; Wang, E.; Dai, H. J., Highly conducting graphene sheets and Langmuir-Blodgett films. Nat Nanotechnol 2008, 3 (9), 538-542; (b) Blake, P.; Brimicombe, P. D.; Nair, R. R.; Booth, T. J.; Jiang, D.; Schedin, F.; Ponomarenko, L. A.; Morozov, S. V.; Gleeson, H. F.; Hill, E. W.; Geim, A. K.; Novoselov, K. S., Graphene-based liquid crystal device. Nano Lett 2008, 8 (6), 1704-1708. 
13. (a) Balandin, A. A., Thermal properties of graphene and nanostructured carbon materials. Nat Mater 2011, 10 (8), 569-581; (b) Seol, J. H.; Jo, I.; Moore, A. L.; Lindsay, L.; Aitken, Z. H.; Pettes, M. T.; Li, X. S.; Yao, Z.; Huang, R.; Broido, D.; Mingo, N.; Ruoff, R. S.; Shi, L., TwoDimensional Phonon Transport in Supported Graphene. Science 2010, 328 (5975), 213-216; (c) Renteria, J. D.; Nika, D. L.; Balandin, A. A., Graphene Thermal Properties: Applications in Thermal Management and Energy Storage. Appl Sci-Basel 2014, 4 (4), 525-547.

14. (a) Hesjedal, T., Continuous roll-to-roll growth of graphene films by chemical vapor deposition. Appl Phys Lett 2011, 98 (133106); (b) Bae, S.; Kim, H.; Lee, Y.; Xu, X. F.; Park, J. S.; Zheng, Y.; Balakrishnan, J.; Lei, T.; Kim, H. R.; Song, Y. I.; Kim, Y. J.; Kim, K. S.; Ozyilmaz, B.; Ahn, J. H.; Hong, B. H.; Iijima, S., Roll-to-roll production of 30-inch graphene films for transparent electrodes. Nat Nanotechnol 2010, 5 (8), 574-578.

15. Rafiee, J.; Mi, X.; Gullapalli, H.; Thomas, A. V.; Yavari, F.; Shi, Y. F.; Ajayan, P. M.; Koratkar, N. A., Wetting transparency of graphene. Nat Mater 2012, 11 (3), 217-222.

16. (a) Shih, C. J.; Wang, Q. H.; Lin, S. C.; Park, K. C.; Jin, Z.; Strano, M. S.; Blankschtein, D., Breakdown in the Wetting Transparency of Graphene. Phys Rev Lett 2012, 109 (176101); (b) Shih, C. J.; Strano, M. S.; Blankschtein, D., Wetting translucency of graphene. Nat Mater 2013, $12(10), 866-869$.

17. (a) Schrader, M. E., Ultrahigh-Vacuum Techniques in Measurement of Contact Angles .3. Water on Copper and Silver. J Phys Chem-Us 1974, 78 (1), 87-89; (b) Trevoy, D. J.; Johnson, H., The Water Wettability of Metal Surfaces. J Phys Chem-Us 1958, 62 (7), 833-837.

18. (a) Bewig, K. W.; Zisman, W. A., Wetting of Gold and Platinum by Water. J Phys ChemUs 1965, 69 (12), 4238-4242; (b) Preston, D. J.; Miljkovic, N.; Sack, J.; Enright, R.; Queeney, J.; Wang, E. N., Effect of hydrocarbon adsorption on the wettability of rare earth oxide ceramics. Appl Phys Lett 2014, 105 (11601); (c) Schrader, M. E., Wettability of Clean Metal-Surfaces. J Colloid Interf Sci 1984, 100 (2), 372-380; (d) Schrader, M. E., Ultrahigh-Vacuum Techniques in Measurement of Contact Angles .2. Water on Gold. J Phys Chem-Us 1970, 74 (11), 2313-2317; (e) Bernett, M. K.; Zisman, W. A., Confirmation of Spontaneous Spreading by Water on Pure Gold. J Phys Chem-Us 1970, 74 (11), 2309-2312.

19. (a) Umezu, I.; Kohno, K.; Aoki, K.; Kohama, Y.; Sugimura, A.; Inada, M., Effects of argon and hydrogen plasmas on the surface of silicon. Vacuum 2002, 66 (3-4), 453-456; (b) Nickerson, R., Plasma surface modification for cleaning and adhesion. Polymers, Laminations \& 
Coatings Conference, Books 1 and 2 1998, 1101-1108; (c) Wasy, A.; Balakrishnan, G.; Lee, S. H.; Kim, J. K.; Kim, D. G.; Kim, T. G.; Song, J. I., Argon plasma treatment on metal substrates and effects on diamond-like carbon (DLC) coating properties. Crystal Research and Technology 2014, 49 (1), 55-62.

20. (a) Erb, R. A., Wettability of Metals under Continuous Condensing Conditions. J Phys Chem-Us 1965, 69 (4), 1306-1309; (b) Wilkins, D. G.; Bromley, L. A.; Read, S. M., Dropwise and Filmwise Condensation of Water Vapor on Gold. Aiche J 1973, 19 (1), 119-123; (c) Woodruff, D. W.; Westwater, J. W., Steam Condensation on Electroplated Gold - Effect of Plating Thickness. Int J Heat Mass Tran 1979, 22 (4), 629-632.

21. (a) Bhaviripudi, S.; Jia, X. T.; Dresselhaus, M. S.; Kong, J., Role of Kinetic Factors in Chemical Vapor Deposition Synthesis of Uniform Large Area Graphene Using Copper Catalyst. Nano Lett 2010, 10 (10), 4128-4133; (b) Li, X. S.; Cai, W. W.; An, J. H.; Kim, S.; Nah, J.; Yang, D. X.; Piner, R.; Velamakanni, A.; Jung, I.; Tutuc, E.; Banerjee, S. K.; Colombo, L.; Ruoff, R. S., Large-Area Synthesis of High-Quality and Uniform Graphene Films on Copper Foils. Science 2009, 324 (5932), 1312-1314; (c) Reina, A.; Jia, X. T.; Ho, J.; Nezich, D.; Son, H. B.; Bulovic, V.; Dresselhaus, M. S.; Kong, J., Large Area, Few-Layer Graphene Films on Arbitrary Substrates by Chemical Vapor Deposition. Nano Lett 2009, 9 (1), 30-35.

22. Nair, R. R.; Blake, P.; Grigorenko, A. N.; Novoselov, K. S.; Booth, T. J.; Stauber, T.; Peres, N. M. R.; Geim, A. K., Fine structure constant defines visual transparency of graphene. Science 2008, 320 (5881), 1308-1308.

23. (a) Gao, L. C.; McCarthy, T. J., Wetting 101 degrees. Langmuir 2009, 25 (24), 1410514115; (b) Gao, L. C.; McCarthy, T. J., Contact angle hysteresis explained. Langmuir 2006, 22 (14), 6234-6237.

24. (a) Miljkovic, N.; Enright, R.; Wang, E. N., Modeling and Optimization of Superhydrophobic Condensation. Journal of Heat Transfer 2013, 135 (11), 111004-111004; (b) Kim, S.; Kim, K. J., Dropwise Condensation Modeling Suitable for Superhydrophobic Surfaces. Journal of Heat Transfer 2011, 133 (8), 081502-1 - 081502-7; (c) Rose, J. W.; Glicksman, L. R., Dropwise condensation - the distribution of drop sizes. Int J Heat Mass Tran 1973, 16, 411-425; (d) Rumaa, J., Drop Size Distribution and Heat-Flux of Dropwise Condensation. Chem Eng J Bioch Eng 1978, 16 (3), 171-176. 
25. Lam, C. N. C.; Wu, R.; Li, D.; Hair, M. L.; Neumann, A. W., Study of the advancing and receding contact angles: liquid sorption as a cause of contact angle hysteresis. Adv Colloid Interfac 2002, 96 (1-3), 169-191.

26. (a) Tanner, D. W.; Pope, D.; Potter, C. J.; West, D., Heat Transfer in Dropwise Condensation at Low Steam Pressures in Absence and Presence of Non-Condensable Gas. Int J Heat Mass Tran 1968, 11 (2), 181-182; (b) Sundararaman, T. G.; Venkatram, T., Heat-Transfer during Dropwise Condensation of Steam in Presence of Non-Condensable Gases - Effects of Geometrical Shape of Surface Reversal of Cooling Water-Flow and Orientation. Indian J Technol 1976, 14 (7), 313-321.

27. (a) Vosough, A.; Falahat, A.; Vosough, S.; Esfehani, H. n.; Behjat, A.; Rad, R. n., Improvement Power Plant Efficiency with Condenser Pressure. INTERNATIONAL JOURNAL OF MULTIDISCIPLINARY SCIENCES AND ENGINEERING 2011, 2 (3), 38-43; (b) Bejan, A., Fundamentals of exergy analysis, entropy generation minimization, and the generation of flow architecture. Int J Energ Res 2002, 26 (7), 545-565.

28. Wilmshurst, R.; Rose, J. W., Dropwise Condensation-Further Heat Transfer Measurements. In Fourth International Heat Transfer Conference, Elsevier: Paris-Versailles, France, 1970; Vol. 6.

29. Leidheiser, H., Corrosion of Painted Metals - a Review. Corrosion 1982, 38 (7), 374-383.

30. Shahil, K. M. F.; Balandin, A. A., Graphene-Multilayer Graphene Nanocomposites as Highly Efficient Thermal Interface Materials. Nano Lett 2012, 12 (2), 861-867.

31. (a) Boreyko, J. B.; Chen, C. H., Vapor chambers with jumping-drop liquid return from superhydrophobic condensers. Int J Heat Mass Tran 2013, 61, 409-418; (b) Boreyko, J. B.; Chen, C. H., Self-Propelled Dropwise Condensate on Superhydrophobic Surfaces. Phys Rev Lett 2009, 103 (18), 184501-1 - 184501-4; (c) Miljkovic, N.; Enright, R.; Nam, Y.; Lopez, K.; Dou, N.; Sack, J.; Wang, E. N., Jumping-Droplet-Enhanced Condensation on Scalable Superhydrophobic Nanostructured Surfaces. Nano Lett 2013, 13 (1), 179-187; (d) Miljkovic, N.; Wang, E. N., Condensation heat transfer on superhydrophobic surfaces. Mrs Bull 2013, 38 (5), $397-406$. 